\title{
Morphotypological characteristics of primary school children engaged in karate Kyokushin
}

\author{
Irina Kalinina*, Yulia Dyakova, Marina Polovnikova, Yulia Kudryashova, Marina Polovnikova \\ ${ }^{1}$ Kuban State University of Physical Culture, Sports and Tourism, 350015, Krasnodar, Russia
}

\begin{abstract}
We use anthropometric methods and caliperometry to determine the features of the primary school children physique type involved in Kyokushinkai karate, and to identify the morphological selection criteria at the stage of sports development, the morphotypological indicators of 28 primary school children were studied ( 8 boys at the age of 7 years and 20 boys in age 10). The length and weight of the body, total, partial body dimensions, as well as the thickness of the subcutaneous fat folds were determined. Fat-free body mass, fat, muscle and bone components were calculated by the analytical method proposed by J. Matejko. Body proportions were established according to the classification of P.N. Bashkirov based on the percentage of the longitudinal and transverse dimensions of the body to its length. The somatotype was determined by the Heath-Carter method and was established on the basis of anthropometric calculation formulas. The obtained data characterizing the peculiarities of physical development, taking into account age and in the course of sports. Differences in the overall, component composition of the body in children of different ages under the influence of physical activity have been established. For children of primary school age (7 and 10 years old) who engage in Kyokushinkai karate, meso-ectomorphic and ecto-mesomorphic body types are characteristic. It is expressed in well-developed muscles and insignificant fat deposition with sufficient elongation of the body.
\end{abstract}

\section{Introduction}

Karate for children is a system of general physical and spiritual development, which contains elements of martial art. Karate strengthens the body, develops motor skills, strength, endurance, hardens the will, contributes to the spiritual and moral development, self-improvement of the child. However, at the same time, high achievements in sports from an early age, in addition to skill, largely depend on body size, body type and body composition of the athlete. During the intensive training process, changes occur in the child's body, leading to the formation of a certain morphotype characteristic of this specialization. During the diagnosis of the morphological parameters of children-athletes and the establishment of their body types, some difficulties arise, since the morphotype of most of them does not correspond to the established typological values adopted in one or another specialization. Whereas, the morphological type is one of the most important informative indicators characterizing the state of health and development of the body [1,2]. The use of data on the morphotype and systematic observations of children involved in karate, in combination with the data of other studies, will make it possible to more accurately assess the impact of the assigned training load, the dynamics of recovery processes, the degree of physical performance, as well as the functional state of the child as a whole. Karate Kyokushinkai is a mixed sport. During training, there is a frequent change in sports working posture, both halves of the athlete's body experience constantly changing symmetrical and asymmetric loads. The location of the spine also changes many times, there is no forced sports posture, and if it occurs, it is short-term. The muscles of the trunk, abdomen and limbs develop evenly [3]. Therefore, the more fully the training will correspond to the individual characteristics of the development of children, the faster they will progress and reach great heights in sports. In addition, information from literary sources on this topic is rather fragmentary, many data cannot be used for comparative assessment, since they were obtained several years ago, and during this time the methodology of training athletes has changed significantly, bit standards and record levels have increased.

\section{Materials and methods}

All studies were carried out in compliance with the bioethical requirements for conducting research, based on the recommendations of the 1964 Declaration of Helsinki. The first study involved 28 primary school children involved in Kyokushinkai karate. There are 8 boys aged 7-8 years and 20 boys aged 9-10 years. The surveyed children were characterized by an average social status of the family, approximately the same type and balance of nutrition, the

\footnotetext{
* Corresponding author: kalininirina@yandex.ru
} 
number and duration of training processes, and the absence of chronic diseases. In order to obtain data on the morphotypological status of the studied categories of children, informed consent was obtained from parents for examination. The work used anthropometric, caliperometric methods for determining body composition [4]. The study consisted in determining the length and weight of the body, total, partial body dimensions, as well as the thickness of the subcutaneous fat folds. Fat-free body weight, fat, muscle and bone components were determined by the analytical method proposed by J. Matejko. Body proportions were established according to the classification of P.N. Bashkirov based on the percentage of the longitudinal and transverse dimensions of the body to its length [4]. The somatotype by the Heath-Carter method was established on the basis of anthropometric calculation formulas [4]. Statistical data processing was performed using the STATISTICA program, the significance of the differences was discussed at the 5\% significance level.

\section{Results and discussion}

We used the anthropometric approach in the study of the physical development of primary school children involved in Kyokushinkai karate, data on the component and overall body composition of this age group were obtained. Evaluation of all the studied parameters revealed a positive dynamic of their increase as children grow up. It was found that the average body length in 8-year-old boys was $122.13 \pm 5.9 \mathrm{~cm}$ in the age group of $9-10$ years, this indicator varied in the range $-142.8 \pm 3.54 \mathrm{~cm}$. At the same time, the coefficients of variation in different ages are different. So, the minimum coefficient of variation among young athletes was noted at 10 years old $(\mathrm{V}=2.48 \%)$, and the maximum - at 7 years old $(4.83 \%)$. It was revealed that the dynamics of body weight gain has a smooth increasing trend with a maximum absolute value of 10 years. The level of within-group variation in body weight is higher. The range of body weight values in the group of 7-8-year-old boys ranged from 16.0 to $22.0 \mathrm{~kg}$ with a minimum coefficient of variation $(\mathrm{V}=9.85 \%)$. Whereas in 9-10-year-old boys this indicator ranged from 22.0 to $55.0 \mathrm{~kg}$, and the coefficient of variation was $\mathrm{V}=21.6 \%$. When assessing the values of lean body mass in young athletes, no statistically significant differences were found. When assessing the individual indicators of boys, it was revealed that at the age of seven it was on average $97.8 \%$, and at the age of 10 it was $95.7 \%$. The analysis of changes in the composition of the body in terms of age showed their different distribution and dynamics of development (Fig. 1). The highest content in the body structure was revealed for the muscle component. So, for children of 10 years old, it averaged $49.7 \%$, and for younger athletes this indicator was lower by $6.3 \%$. The study of data on the development of subcutaneous fat deposition showed (Fig. 1) that 9-10-year-old boys in an older state have the least results in terms of adipose tissue content (8.3\%) compared to other body components, while for athletes more in the younger age group, this parameter was quite high $(25.3 \%)$. The study of the distribution of the fat component revealed the relative uniformity of its distribution with predominant accumulation in the abdomen, on the back of the thigh and shoulder. Equal, smooth growth of the bone component composition is noted as they grow older (Fig. 1). In the group of 9-10-year-old boys, who engaged in for Kyokushinkai karate, this indicator was higher by $10.6 \%$ compared to the data of the age group of young karate fighters 7-8 years old.

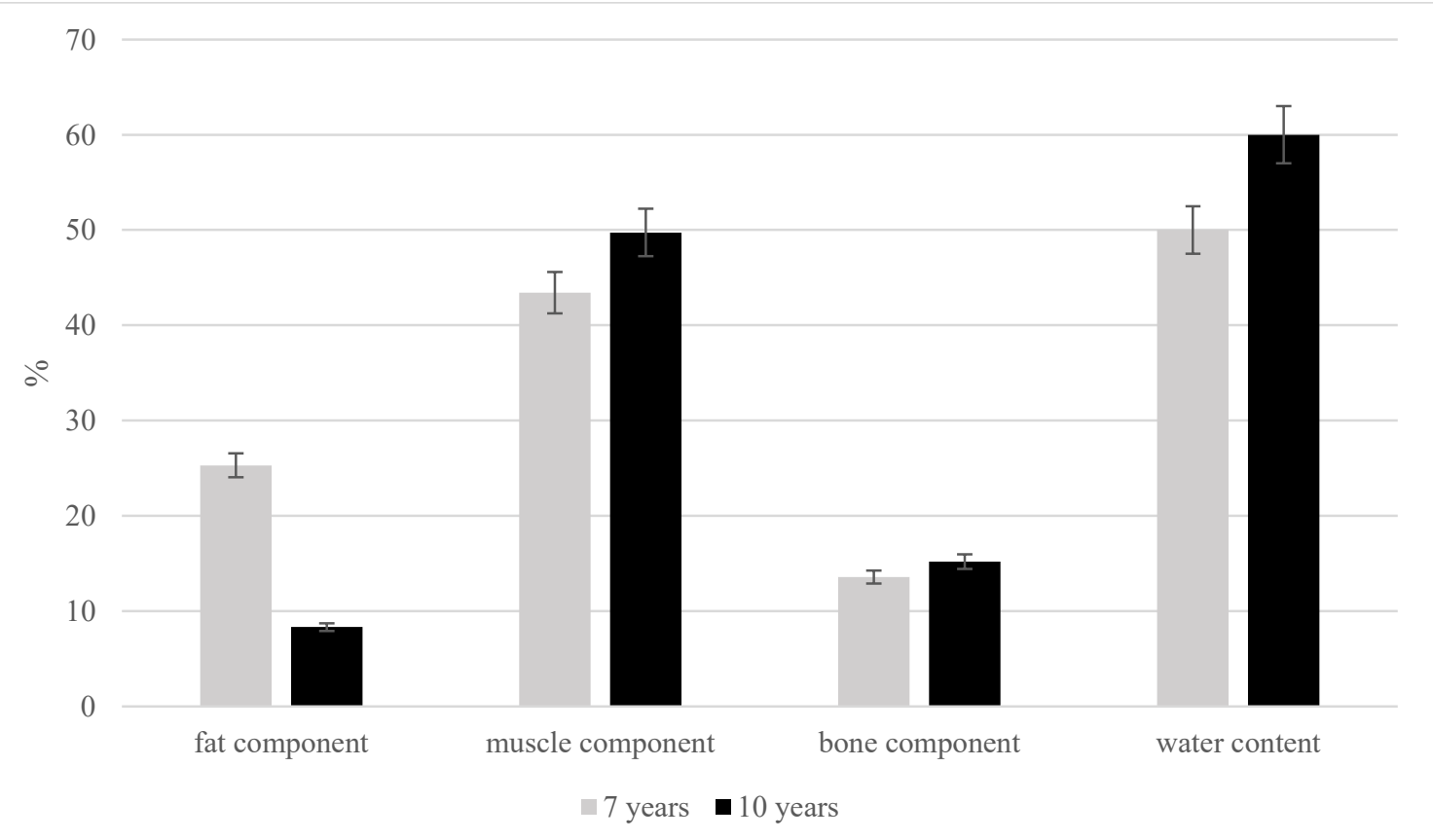

Fig. 1. Component composition of body weight of primary school children engaged in Kyokushin karate.

In the human body, water plays a dominant role, since in the body itself, water makes up $65-75 \%$ of the total body weight, all the most important biochemical reactions occur directly with the participation of water. In the course of the work, it was found (Fig. 1) that as boys grew older, the total water content in their body increased. If this parameter 
reached $50 \%$ for young karate athletes aged $7-8$, it was $10 \%$ higher for $9-10$-year-old athletes. This difference in the total water content is explained by the fact that at the early stages of children's development, there is a rather high tension and instability of water-salt metabolism, which are determined by the intensive growth of the child and the relative immaturity of the neuroendocrine and renal regulation systems [5]. In addition, there is a close correlation between the total water content and the fat content, that is, the higher the fat content, the lower the water content, and, conversely, the higher the percentage of active body weight, the higher the water content. This relationship was also established during the analysis of the component composition of the body of primary school children engaged in Kyokushin karate.

Analysis of the proportionality of the body according to the classification of N. p. Bashkirov showed that half of the examined young karate athletes aged 9-10 years have a dolichomorphic type of body structure (Fig. 2), that is, they were characterized by a narrow elongated body, long limbs and a short trunk. A small part (5\%) is represented by mesomorphic subjects, which are characterized by averaged values of anatomical features of the body structure. Also, a fairly large group (45\%) has a transitional type - dolicho-mesomorphic.

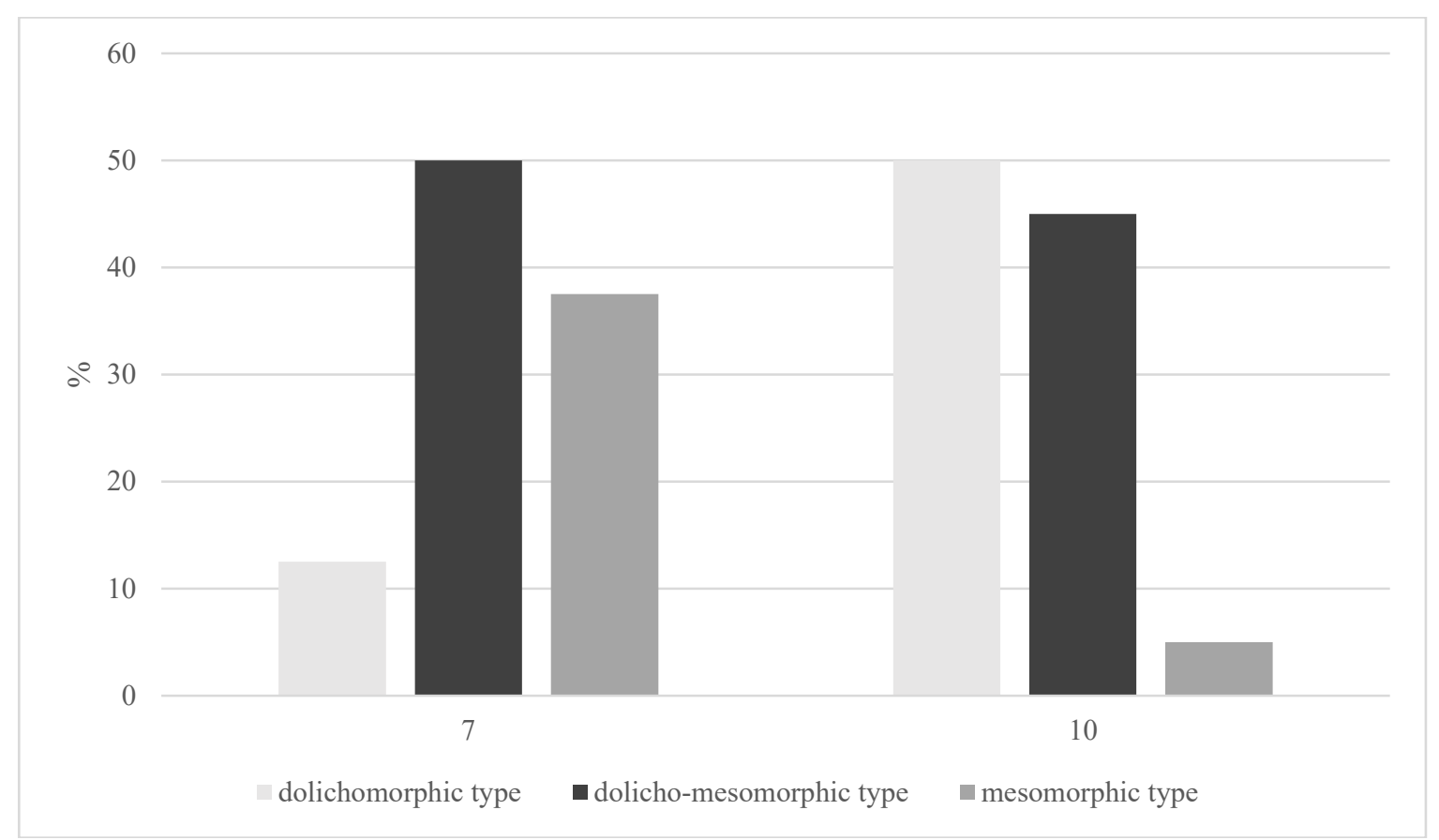

Fig. 2. Types of proportions of the body of primary school children engaged in Kyokushinkai karate.

There is an opinion that the total weight and body composition can be changed with the help of targeted physical training, directly creating an athlete's physique corresponding to the model characteristics [7-8]. The characteristic of the somatotype proposed by B. Heath and D. Carter is of considerable interest in the study of morphological and functional characteristics of athletes. Somatotyping according to the Heath-Carter method takes into account three components that reflect the characteristics of the physique: the level of development of adipose (endomorphic), bone and muscle (mesomorphy) tissues and the measure of elongation of the body (ectomorphy) [4]. Somatotyping based on anthropometric data is considered the most accurate [9-11], since a large number of the human body morphological parameters measurements are carried out and only then, on the basis of formula calculations, a conclusion is made about belonging to a particular type of physique. When determining the Heath-Carter somatotype in young karate athletes, it was found that for boys aged 7-8 years, the meso-ectomorphic somatotype is characteristic, while at 9-10 years old, the ecto-mesomorphic type is predominant among children.karate Fighters of an ecto-mesomorphic body type with high mesomorphic indices, the values of ectomorphism are significantly higher than that of endomorphism, which indicates the elongation of the body and its segments in comparison with representatives of the balanced mesomorphic type.

\section{Conclusions}

The study of morphotypological indicators of children of primary school age, who engaged in for Kyokushinkai karate, using the anthropometric method made it possible to obtain data that characterized the features of physical development, taking into account age and during the course of this sport. Differences in the overall, component composition of the body were revealed in children of different ages under the influence of physical activity. Boys aged 9-10 years compared with 7-8-year-olds are characterized by higher skeletal massiveness. It is confirmed by high values of bone and muscle tissue. The predominance of the muscle component in young athletes of this specialization is explained by adaptation to training loads, while the fat component remains within the normal range. For children of primary school age (7-10 years old), who involved in for Kyokushinkai karate, meso-ectomorphic and ectomesomorphic body types are characteristic. It is expressed in well-developed muscles and insignificant fat deposition 
with a sufficient elongation of the body. The use of the obtained indicators during systematic observations of young athlete's in combination with the data of other studies will allow to more accurately assess the impact of the assigned training load, as well as the functional state of athletes to achieve high sports results

\section{References}

1. E.A. Oleynik, Scientific notes of the P.F. Lesgaft University, 3(121), 97-101 (2015)

2. V.V. Grebennikova, Siberian medical journal, 3, 78-82 (2003)

3. A.S. Kuznetsov, Z.M. Kuznetsova, Russian Journal of Physical Education and Sport, 14(4), 5-7 (2019). DOI: 10.14526/20704798-2019-14-4-5-7

4. G.D. Aleksanyants, Age and sports morphology: educational and methodological guide (KSUFKST, Krasnodar, 2017)

5. A.A. Baranov, V.R. Kuchma, N.A. Skoblina, Physical development of children and adolescents at the turn of the Millennium (Publisher Scientific center for children's health RAMS, M., 2008)

6. A.V. Ageikin, Young scientist, 12, 381-383 (2014)

7. B.A. Nikityuk, Integration of knowledge in science and man: (modern integrative anthropology) (Sportakadempress, M., 2000)

8. M.G. Polovnikova, A.A. Kilimnik, Physical culture and sport. Olympic education, 321-322 (2019)

9. I. Pashkova, I. Gaivoronsky, D. Nikityuk, Somatotype and component composition of the adult body (Spetslit, SPb., 2019)

10. M.G. Tkachuk, A.A. Sobolev, Scientific notes of the P.F. Lesgaft University, 4(134), 282-285 (2016)

11. A.N. Gerasevich, V.A. Kuzmin, E.G. Parkhots, Modern problems of health formation and promotion (HEALTH-2019): collection of scientific articles (Publishing house of the Brest State Technical University, Brest, 34-41, 2019) 